Jul 2001

KEK-TH-778

\title{
Melvin Background in Heterotic Theories
}

\author{
Takao Suyama f \\ Theory Group, KEK \\ Tsukuba, Ibaraki 305-0801, Japan
}

\begin{abstract}
We give an interpretation of the non-supersymmetric heterotic theories as the supersymmetric heterotic theories on Melvin background with Wilson lines. The coincidence of the partition functions are shown for most of the non-supersymmetric theories. We also discuss the tachyonic instability in terms of the non-trivial background fields. The behavior in the strong coupling region is investigated by using the duality relations of the supersymmetric theories.
\end{abstract}

\footnotetext{
${ }^{1}$ e-mail address : tsuyama@post.kek.jp
} 


\section{Introduction}

To understand the structure of string theory, one has to know its vacuum state. Although there are infinitely many perturbative vacua, some of which resemble our world, little is known what the vacuum is nonperturbatively. To analyze the vacuum we need more sophisticated understanding of the nonpertrbative dynamics of the theory, and this problem seems to be still beyond our reach. One possible approach to this problem would be to construct the theory nonperturbatively [1].

It is necessary to understand the (in)stability of the vacua. Recent reseaches originated by Sen [2] have clarified the stabilization mechanism of perturbative instabilities, associated with open string tachyons. This is closely related to the instability of D-branes, and the stabilization corresponds to the decay of the D-branes. The open string field theory [3] plays a crucial role in the investigation of the tachyon condensation [4].

There is another perturbative instability due to closed string tachyons. An interesting conjecture was made in [5] [6] (see also [7]), suggesting that the endpoint of the closed string tachyon condensation in Type 0A theory can be interpreted as the supersymmetric vacuum. This theory is interpreted as Type IIA theory on the Melvin background [8] which breaks all the supersymmetries. And it is also shown that the tachyonic instability in Type 0A theory can be related to the instability due to pair creations of D-branes. The above results might indicate that Type 0A theory decays into Type IIA theory after the closed string tachyon condensation.

In our previous paper [9], we tried to apply a similar interpretation to the nonsupersymmetric heterotic theories [10] 11] 12], and some of them can be understood as the supersymmetric theories on the Melvin background. This would suggest that such understanding of the closed string tachyons is a general one, not specific to Type 0A theory.

In this paper, we extend our investigations to all of the non-supersymmetric heterotic theories in ten dimensions. Although there is one exception, the other theories can be related to the supersymmetric heterotic theories. So this result leads us to a conjecture that the non-supersymmetric heterotic theories would decay into the supersymmetric ones. We also argue the instability due to the closed string tachyons in terms of the background fields in the supersymmetric theories.

Since the non-supersymmetric theories can be related to the supersymmetric ones, one can discuss the strong coupling behavior of the former by using the duality relations of the latter. This enable us to see the validity of the decay picture beyond the perturbation theory.

This paper is organized as follows. We briefly review the properties of the Melvin background (section 2) and the correspondence between Type 0A theory and Type IIA theory stated above (section 3). In section 4, we recall some facts about the non-supersymmetric heterotic theories. They are related to the supersymmetric theories in section 5 by showing the coincidence of the partition functions. The tachyonic instability is interpreted in terms of the background fields in the supersymmetric theories in section 6 . In section 7 , 
we discuss the strong coupling behavior of the non-supersymmetric theories. Section 8 is devoted to discussions. The stabilization of the heterotic $E_{8}$ theory, the exceptional case, is also argued. The explicit expressions of various partition functions discussed in section 5 are summarized in the appendix.

\section{Kaluza-Klein Melvin background}

Consider the following metric in M-theory [5]

$$
d s_{11}^{2}=\eta_{\alpha \beta} d x^{\alpha} d x^{\beta}+d r^{2}+r^{2}(d \theta+q d y)^{2}+d y^{2} .
$$

The indices $\alpha, \beta$ run from 0 to 7 , and $q$ is a real parameter. We have used the polar coordinates $r, \theta$ in the 8-9 plane. The $y$-direction is compactified on $S^{1}$ with the radius $R$.

By introducing the new coordinate $\tilde{\theta}=\theta+q y$, the metric (2.1) becomes the flat one, although the periodicity of $\tilde{\theta}$ is nontrivial,

$$
\begin{aligned}
& y \sim y+2 \pi m R, \\
& \tilde{\theta} \sim \tilde{\theta}+2 \pi n+2 \pi m q R,
\end{aligned}
$$

where $m, n$ are integers. Since the metric (2.1) is locally flat, this is a solution of the Einstein equation. This would still be a solution even if higher derivative terms are included, so this background is expected to be an exact solution of M-theory.

The metric (2.1) describes a nontrivial spacetime in view of the lower dimensions. The dimensional reduction along the $S^{1}$ produces the following ten-dimensional (Type IIA) background in the string frame

$$
\begin{aligned}
d s_{10}^{2} & =\sqrt{1+q^{2} r^{2}}\left(\eta_{\alpha \beta} d x^{\alpha} d x^{\beta}+d r^{2}\right)+\frac{r^{2}}{\sqrt{1+q^{2} r^{2}}} d \theta^{2} \\
e^{\frac{4}{3} \phi} & =1+q^{2} r^{2} \\
A_{\theta} & =\frac{q r^{2}}{1+q^{2} r^{2}} .
\end{aligned}
$$

The dilaton diverges as $r \rightarrow \infty$, so this background cannot be analyzed by the perturbation theory. In addition, from (2.6) there is a nonzero component of the R-R field strength

$$
F_{89}=\frac{2 q}{\left(1+q^{2} r^{2}\right)^{2}}
$$

which makes the perturbaive analysis harder. Therefore, the background (2.6) describes a localized R-R flux around $r=0$ with the total flux $1 / q$. This R-R flux configuration 
is called a fluxbrane or a F7-brane [5] 6]. Further investigations on the fluxbranes can be found in [13] [14].

From the identification (2.3), one can see the periodicity in $q$. If $q=k / R$ for some integer $k, \tilde{\theta}$ have the period $2 \pi$ and the spacetime is nothing but a direct product $\mathbf{R}^{10} \times S^{1}$, which is the same as that with $q=0$. Thus the parameter $q$ has the period $1 / R$. This periodicity cannot be seen from the ten-dimensional background (2.6).

When there exist spacetime fermions, the situation is more complicated. Suppose the case $q=1 / R$. For the fermions to exist, the boundary condition along the $S^{1}$ should be periodic. Now the parallel transport of the fermions along the $S^{1}$ is accompanied by the

$2 \pi$ shift of $\tilde{\theta}$, so this gives an extra sign. In other words, although the metric is the same, the spin structure is different between $q=0$ and $q=1 / R$. Therefore the period of $q$ should be $2 / R$, when the spin structure is taken into account.

The Melvin background is unstable. The instability is investigated in [15], and it is due to pair creations of D6-branes in the Type IIA picture, which is a similar phenomenon to the Schwinger pair creation in an electric field. It is shown in [15] that the decay mode is described by the Myers-Perry Kerr instanton [16], and the decay late is zero only at $q=0$. So this result indicates that the Melvin background decays into the KaluzaKlein background without R-R flux. On the other hand, as mentioned above, the Melvin background with $q=1 / R$ (we will call it "critical" Melvin) can be interpreted as the Kaluza-Klein background with the twisted boundary condition for fermions along the $S^{1}$. In this picture, the instability of this background is described by an expanding bubble [17], corresponding to the Schwarzchild instanton. It is shown in [17] that the Kaluza-Klein background is unstable against the nucleation of such bubbles, unless the spin structure is suitably chosen, i.e. a fermion can exist on the background. For the critical Melvin, the boundary condition is antiperiodic due to the twisting, so this background is unstable.

\section{IIA-0A duality}

It is discussed in [18] that M-theory compactified on the twisted $S^{1}$ is equivalent to Type 0A theory, just like the ordinary $S^{1}$ compactification is equivalent to Type IIA theory. The evidence of this equivalence is the following.

Compactify the background (2.1) further on $S^{1}$, say, along the 6-direction, and take the radius of the $S^{1}$ to be zero. Then the resulting ten-dimensional background is the Kaluza-Klein Melvin background without R-R flux, and the dilaton is constant. So this background can be analyzed by the perturbative Type IIA theory.

One-loop partition function can be calculated exactly [19]

$$
Z_{q}=\int \frac{d^{2} \tau}{\tau_{2}} \tau_{2}^{-4}|\eta(\tau)|^{-18} \sum_{m, n \in \mathbf{Z}} \exp \left[-\frac{\pi R^{2}}{\alpha^{\prime} \tau_{2}}|n+m \tau|^{2}\right]
$$




$$
\times\left|\vartheta\left[\begin{array}{c}
1 / 2+m q R \\
1 / 2+n q R
\end{array}\right](0, \tau)\right|^{-2}\left|\vartheta\left[\begin{array}{c}
1 / 2+m q R / 2 \\
1 / 2+n q R / 2
\end{array}\right](0, \tau)\right|^{8}
$$

We have used the theta function with characteristics

$$
\vartheta\left[\begin{array}{l}
a \\
b
\end{array}\right](\nu, \tau)=\sum_{n \in \mathbf{Z}} \exp \left[\pi i(n+a)^{2} \tau+2 \pi i(n+a)(\nu+b)\right] .
$$

From the expression (3.1) one can see the periodicity in $q$ as the periodicity of the theta functions. The period is $2 / R$ since Type IIA theory contains spacetime fermions. At $q=1 / R$ one finds

$$
Z_{1 / R} \sim \int \frac{d^{2} \tau}{\tau_{2}} \tau_{2}^{-5}|\eta(\tau)|^{-16}\left(\left|Z_{0}^{0}(\tau)\right|^{8}+\left|Z_{1}^{0}(\tau)\right|^{8}+\left|Z_{0}^{1}(\tau)\right|^{8}\right)
$$

if $R$ is sufficiently small. The RHS coincides with the partition function for Type 0A theory compactified on $S^{1}$ with vanishing radius. Thus we find that Type IIA theory on the critical Melvin and Type 0A theory both have the same mass spectrum in a certain limit.

Suppose that this correspondence holds even before the dimensional reduction along the 6-direction. Then the above result suggests that M-theory on the twisted $S^{1}$, which is equivalent to M-theory on the critical Melvin, is equivalent to Type 0A theory.

According to the above interpretation of Type 0A theory, it is expected that the instability of the Melvin background (or of the Kaluza-Klein background without fermions) would correspond to the one due to the closed string tachyon in Type 0A theory [5] 6]. The stabilization of closed string tachyons seems to be a more difficult problem to deal with than that of open string tachyons [2]. So the above-mentioned interpretation would give us an insight into the closed string tachyon condensation.

\section{Heterotic theories in ten dimensions}

Type 0A theory discussed in the previous section can be regarded as a Type IIA theory twisted by a discrete goup $\mathbf{Z}_{2}$. This $\mathbf{Z}_{2}$ is generated by $(-1)^{F_{s}}$ where $F_{s}$ is the spacetime fermion number. Such a procedure enables us to construct a new string theory from the known one. This was investigated for the heterotic theories in [10] 11], and several ten-dimensional heterotic theories were discovered. And finally, the list of the heterotic theories in ten dimensions was completed in [12]. There are nine theories, two of which are the well-known supersymmetric theories. Among the remaining seven, which have

no supersymmetry, only one theory is tachyon-free, while the others contain tachyons in their mass spectrum. The non-supersymmetric theories can be characterized by their gauge groups as follows. 
1. The $S O(16) \times S O(16)$ theory without any tachyon.

2. The $S O(32)$ theory with tachyons in the $\mathbf{3 2}$ representation of the gauge group.

3. The $E_{8} \times S O(16)$ theory with tachyons in the $(\mathbf{1}, \mathbf{1 6})$.

4. The $S O(8) \times S O(24)$ theory with tachyons in the $\left(\mathbf{8}_{\mathbf{s}}, \mathbf{1}\right)$.

5. The $E_{7} \times S U(2) \times E_{7} \times S U(2)$ theory with tachyons in the $(\mathbf{1}, \mathbf{2}, \mathbf{1}, \mathbf{2})$.

6 . The $U(16)$ theory with two tachyons in the singlet.

7. The $E_{8}$ theory with only one tachyon.

As shown in [10], each theory, except for the $E_{8}$ theory, can be constructed from the supersymmetric theories by a $\mathbf{Z}_{2}$ twist which is generated by $(-1)^{F_{s}} \gamma_{\delta}$, where $\gamma_{\delta}$ is a shift operator in the internal momentum space, in view of the bosonic construction of the current algebra, with shift vector $\delta$. Of course, the $\gamma_{\delta}$ satisfies $\gamma_{\delta}^{2}=1$.

Consider an example; $\delta=\left(1,0^{15}\right)$. It is convenient to discuss the internal left-moving degrees of freedom in the fermionic representation $\lambda_{L}^{I}(I=1, \cdots, 32)$. Then the action of the $\gamma_{\delta}$ is

$$
\gamma_{\delta}=e^{2 \pi i J_{12}}
$$

where $J_{12}$ is the generator of the $S O(32)$ which acts only on $\lambda_{L}^{1,2}$. This acts trivially on the vector representation of the $S O(32)$ and gives -1 for the spinor representation. Thus the untwisted sectors are

$$
(\text { left, right })=(N S+, N S+) \oplus(R+, R+) .
$$

In the twisted sectors, the $\lambda_{L}^{1,2}$ obey the following boundary conditions

$$
\lambda_{L}^{1,2}(\sigma+2 \pi)=e^{2 \pi i} \lambda_{L}^{1,2}(\sigma)
$$

So this does not seem to change anything. However, the fermion number of the vacuum changes by the spectral flow. Therefore, in the twisted sectors the spectrum is restricted by the opposite GSO projection. The GSO projection of the right-moving sectors is also reversed and we obtain

$$
(N S-, N S-) \oplus(R-, R-) .
$$

This spectrum coincides with that of the non-supersymmetric $S O(32)$ theory. The other constructions will be reviewed briefly in the appendix.

\section{$5 \quad$ Heterotic theories on Melvin background}

In this section, we will calculate partition functions of the supersymmetric heterotic theories on the Melvin background with various patterns of Wilson lines. It can be shown that the partition functions coincide with those of the non-supersymmetric heterotic theories 
reviewed in section 4 , in a certain limit. This fact would be a piece of evidence for the equivalence between the non-supersymmetric heterotic theories and the supersymmetric heterotic theories on the Melvin background.

\subsection{Partition functions}

We consider the supersymmetric heterotic theories on the Melvin background with a general Wilson line. The worldsheet action is as follows,

$$
\begin{aligned}
S & =\frac{1}{4 \pi \alpha^{\prime}} \int d^{2} \sigma\left\{\eta_{\mu \nu} \partial_{a} X^{\mu} \partial_{a} X^{\nu}+\left|\partial_{a} X+i q \partial_{a} Y X\right|^{2}+\left(\partial_{a} Y\right)^{2}\right\} \\
& +\frac{i}{\pi} \int d^{2} \sigma \bar{S}_{R}^{r}\left(\partial_{+}+\frac{i}{2} q \partial_{+} Y\right) S_{R}^{r}+\frac{i}{\pi} \int d^{2} \sigma \sum_{I=1}^{16} \bar{\lambda}_{L}^{I}\left(\partial_{-}-i A_{Y}^{I} \partial_{-} Y\right) \lambda_{L}^{I}
\end{aligned}
$$

where $\mu, \nu=0, \cdots, 6$ and $r=1, \cdots, 4$. We have employed the Green-Schwarz formalism for the right-moving fermions $S_{R}^{r}$, while the left-moving fermions $\lambda_{L}^{I}$ are the RNS fermions. Although this action has nontrivial couplings between $Y$ and other fields, the theory is conformally invariant for any value of $q$ [20].

An interesting property of the action (5.1) is that this can be reduced to a free action [19]. This is accomplished by the following field redefinitions

$$
\begin{aligned}
& X=e^{-i q Y} \tilde{X}, \\
& S_{R}^{r}=e^{-\frac{i}{2} q Y} \tilde{S}_{R}^{r}, \\
& \lambda_{L}^{I}=e^{i A_{Y}^{I} Y} \tilde{\lambda}_{L}^{I} .
\end{aligned}
$$

Note that the Jacobian of this field redefinitions is trivial. The existence of the nontrivial background is now encoded in the boundary conditions for the new fields $\tilde{X}, \tilde{S}_{R}^{r}, \tilde{\lambda}_{L}^{I}$.

We will calculate the partition function on the torus with the modulus $\tau=\tau_{1}+i \tau_{2}$. To do this, we have to specify the boundary conditions. $X^{\mu}$ and $Y$ has the ordinary periodicity,

$$
\begin{aligned}
& X^{\mu}\left(\sigma^{1}+2 \pi, \sigma^{2}\right)=X^{\mu}\left(\sigma^{1}+2 \pi \tau_{1}, \sigma^{2}+2 \pi \tau_{2}\right)=X^{\mu}\left(\sigma^{1}, \sigma^{2}\right), \\
& Y\left(\sigma^{1}+2 \pi, \sigma^{2}\right)=Y\left(\sigma^{1}, \sigma^{2}\right)-2 \pi m R, \\
& Y\left(\sigma^{1}+2 \pi \tau_{1}, \sigma^{2}+2 \pi \tau_{2}\right)=Y\left(\sigma^{1}, \sigma^{2}\right)+2 \pi n R,
\end{aligned}
$$

where $m, n$ are integers. The boundary conditions for the other fields defined in (5.2) are twisted as follows

$$
\begin{aligned}
& \tilde{X}\left(\sigma^{1}+2 \pi, \sigma^{2}\right)=e^{-2 \pi i m q R} \tilde{X}\left(\sigma^{1}, \sigma^{2}\right), \\
& \tilde{X}\left(\sigma^{1}+2 \pi \tau_{1}, \sigma^{2}+2 \pi \tau_{2}\right)=e^{2 \pi i n q R} \tilde{X}\left(\sigma^{1}, \sigma^{2}\right), \\
& \tilde{S}_{R}^{r}\left(\sigma^{1}+2 \pi, \sigma^{2}\right)=e^{-\pi i m q R} \tilde{S}_{R}^{r}\left(\sigma^{1}, \sigma^{2}\right),
\end{aligned}
$$




$$
\begin{aligned}
& \tilde{S}_{R}^{r}\left(\sigma^{1}+2 \pi \tau_{1}, \sigma^{2}+2 \pi \tau_{2}\right)=e^{\pi i n q R} \tilde{S}_{R}^{r}\left(\sigma^{1}, \sigma^{2}\right), \\
& \tilde{\lambda}_{L}^{I}\left(\sigma^{1}+2 \pi, \sigma^{2}\right)= \pm e^{2 \pi i m A_{Y}^{I} R} \tilde{\lambda}_{L}^{I}\left(\sigma^{1}, \sigma^{2}\right), \\
& \tilde{\lambda}_{L}^{I}\left(\sigma^{1}+2 \pi \tau_{1}, \sigma^{2}+2 \pi \tau_{2}\right)= \pm e^{-2 \pi i n A_{Y}^{I} R} \tilde{\lambda}_{L}^{I}\left(\sigma^{1}, \sigma^{2}\right) .
\end{aligned}
$$

The plus (minus) signs in (5.10) (5.11) are the ordinary ones for the R (NS) sector, respectively.

The partition function for a left-moving fermion with a twisted boundary condition is easily calculated, for example, by using the operator formalism (see e.g. 21]). For the fermion with boundary conditions

$$
\begin{aligned}
& \psi\left(\sigma^{1}+2 \pi, \sigma^{2}\right)=-e^{2 \pi i \theta} \psi\left(\sigma^{1}, \sigma^{2}\right), \\
& \psi\left(\sigma^{1}+2 \pi \tau_{1}, \sigma^{2}+2 \pi \tau_{2}\right)=-e^{-2 \pi i \phi} \psi\left(\sigma^{1}, \sigma^{2}\right),
\end{aligned}
$$

the partition function is, up to overall factor,

$$
\begin{aligned}
Z_{2 \phi}^{2 \theta}(\tau) & \equiv \vartheta\left[\begin{array}{l}
\theta \\
\phi
\end{array}\right](0, \tau) \eta(\tau)^{-1} \\
& =e^{2 \pi i \theta \phi} q^{\frac{\theta^{2}}{2}-\frac{1}{24}} \prod_{n=1}^{\infty}\left(1+q^{n+\theta-\frac{1}{2}} e^{2 \pi i \phi}\right)\left(1+q^{n-\theta-\frac{1}{2}} e^{-2 \pi i \phi}\right),
\end{aligned}
$$

where $q=e^{2 \pi i \tau}$. The case of a twisted boson is similar. If $\psi$ is a bosonic field with the same boundary condition, its partition function is $\left|Z_{2 \phi}^{2 \theta}(\tau)\right|^{-2}$.

For the heterotic theories, relative phases of the partition functions for various sectors are relevant. These phases have been fixed in [22] by an anomaly argument. The resulting partition functions for the left-moving fermions are the followings.

$$
\begin{array}{llll}
(\mathrm{AA}) & e^{-\pi i m n\left(a^{I}\right)^{2}} Z_{2 n a^{I}}^{2 m a^{I}}(\tau), & (\mathrm{AP}) & e^{-\pi i m a^{I}\left(n a^{I}+1\right)} Z_{1+2 n a^{I}}^{2 m a^{I}}(\tau) \\
(\mathrm{PA}) & e^{-\pi i m n\left(a^{I}\right)^{2}} Z_{2 n a^{I}}^{1+2 m a^{I}}(\tau), & (\mathrm{PP}) & -i e^{-\pi i m a^{I}\left(n a^{I}+1\right)} Z_{1+2 n a^{I}}^{1+2 m a^{I}}(\tau)
\end{array}
$$

Here (AA) etc. indicate the spin structures on the torus, and we have defined $a^{I}=A_{Y}^{I} R$.

From the above results, one can construct the desired partition function as follows.

$$
\begin{aligned}
Z_{q}\left(A_{Y}^{I}\right)= & \int \frac{d^{2} \tau}{\tau_{2}} \tau_{2}^{-4}|\eta(\tau)|^{-12} \sum_{m, n \in \mathbf{Z}} \exp \left[-\frac{\pi R^{2}}{\alpha^{\prime} \tau_{2}}|n+m \tau|^{2}\right]\left|Z_{1+2 n q R}^{1+2 m q R}(\tau)\right|^{-2} \\
& \exp \left[\operatorname{\pi imn}\left\{(q R)^{2}-\sum_{I=1}^{16}\left(a^{I}\right)^{2}\right\}\right] e^{2 \pi i m q R} Z_{L}^{(m, n)}(\tau) Z_{1+n q R}^{1+m q R}(\tau)^{*^{4}}
\end{aligned}
$$

The contribution $Z_{L}^{(m, n)}(\tau)$ from the left-movers depends on which theory we consider. For the $S O(32)$ theory,

$$
\begin{aligned}
Z_{L}^{(m, n)}(\tau)= & \prod_{I=1}^{16} Z_{2 n a^{I}}^{2 m a^{I}}(\tau)+\prod_{I=1}^{16} e^{-\pi i m a^{I}} Z_{1+2 n a^{I}}^{2 m a^{I}}(\tau) \\
& +\prod_{I=1}^{16} Z_{2 n a^{I}}^{1+2 m a^{I}}(\tau)+\prod_{I=1}^{16} e^{-\pi i m a^{I}} Z_{1+2 n a^{I}}^{1+2 m a^{I}}(\tau),
\end{aligned}
$$


while for the $E_{8} \times E_{8}$ theory,

$$
\begin{aligned}
& Z_{L}^{(m, n)}(\tau) \\
= & \left(\prod_{I=1}^{8} Z_{2 n a^{I}}^{2 m a^{I}}(\tau)+\prod_{I=1}^{8} e^{-\pi i m a^{I}} Z_{1+2 n a^{I}}^{2 m a^{I}}(\tau)+\prod_{I=1}^{8} Z_{2 n a^{I}}^{1+2 m a^{I}}(\tau)+\prod_{I=1}^{8} e^{-\pi i m a^{I}} Z_{1+2 n a^{I}}^{1+2 m a^{I}}(\tau)\right) \\
& \times\left(\prod_{I=9}^{16} Z_{2 n a^{I}}^{2 m a^{I}}(\tau)+\prod_{I=9}^{16} e^{-\pi i m a^{I}} Z_{1+2 n a^{I}}^{2 m a^{I}}(\tau)+\prod_{I=9}^{16} Z_{2 n a^{I}}^{1+2 m a^{I}}(\tau)+\prod_{I=9}^{16} e^{-\pi i m a^{I}} Z_{1+2 n a^{I}}^{1+2 m a^{I}}(\tau)\right) .
\end{aligned}
$$

\subsection{Critical Melvin}

We are interested in the case with a special value of $q$, i.e. $q=1 / R$. In this case, although the boundary condition for spacetime fermions on the $S^{1}$ is twisted, the background geometry is equivalent to the direct product $\mathbf{R}^{9} \times S^{1}$. Therefore it is natural to expect that a supersymmetric heterotic theory on the critical Melvin is equivalent to a nonsupersymmetric heterotic theory, as in the IIA-0A case.

To relate the partition function (5.17) to that of the non-supersymmetric theories, we have to choose the appropriate Wilson lines. These are summarized in the appendix. By the choice of the Wilson lines, the function $Z_{L}^{(m, n)}(\tau)$ has the following periodicity in $m$ and $n$,

$$
Z_{L}^{(m+2, n)}(\tau)=Z_{L}^{(m, n+2)}(\tau)=Z_{L}^{(m, n)}(\tau)
$$

This means that $Z_{L}^{(m, n)}(\tau)$ depends only on $m, n(\bmod 2)$. Thus, for example, $Z_{L}^{(2 m, 2 n)}(\tau)$ is the partition function for untwisted fermions and $Z_{L}^{(2 m+1,2 n)}(\tau)$ is the one for fermions twisted in the $\sigma^{1}$ direction. Another property of the Wilson lines is

$$
\sum_{I=1}^{16}\left(a^{I}\right)^{2}=\text { integer. }
$$

Consider first the case in which the sum (5.21) is an odd integer. Under these conditions, the full partition function (5.17) can be rewritten as follows.

$$
\begin{aligned}
Z_{1 / R}\left(A_{Y}^{I}\right)=\int \frac{d^{2} \tau}{\tau_{2}} \tau_{2}^{-5}|\eta(\tau)|^{-16}\left\{\sum_{k, l \in \mathbf{Z}} \exp \left[-\frac{\pi R^{2}}{\alpha^{\prime} \tau_{2}}|(2 l)+(2 k) \tau|^{2}\right] Z_{L}^{(0,0)}(\tau) Z_{1+2 l}^{1+2 k}(\tau)^{*^{4}}\right. \\
+\sum_{k, l \in \mathbf{Z}} \exp \left[-\frac{\pi R^{2}}{\alpha^{\prime} \tau_{2}}|(2 l+1)+(2 k) \tau|^{2}\right] Z_{L}^{(0,1)}(\tau) Z_{2 l}^{1+2 k}(\tau)^{*^{4}} \\
+\sum_{k, l \in \mathbf{Z}} \exp \left[-\frac{\pi R^{2}}{\alpha^{\prime} \tau_{2}}|(2 l)+(2 k+1) \tau|^{2}\right] Z_{L}^{(1,0)}(\tau) Z_{1+2 l}^{2 k}(\tau)^{*^{4}}
\end{aligned}
$$




$$
\left.+\sum_{k, l \in \mathbf{Z}} \exp \left[-\frac{\pi R^{2}}{\alpha^{\prime} \tau_{2}}|(2 l+1)+(2 k+1) \tau|^{2}\right] Z_{L}^{(1,1)}(\tau) Z_{2 l}^{2 k}(\tau)^{*^{4}}\right\}
$$

We have replaced $\left|Z_{1+2 n q R}^{1+2 m q R}(\tau)\right|^{-2}$ in the eq.(5.17), which is singular at $q=1 / R$, with $\tau_{2}^{-1}|\eta(\tau)|^{-4}$. From the Poisson resummation formula [0],

$$
\sum_{k, l \in \mathbf{Z}} \exp \left[-\frac{\pi R^{2}}{\alpha^{\prime} \tau_{2}}|(2 l+\delta)+(2 k+\epsilon) \tau|^{2}\right]=\mathcal{Z}_{(\epsilon, 0)}+(-1)^{\delta} \mathcal{Z}_{(\epsilon, 1)},
$$

where

$$
\mathcal{Z}_{(\epsilon, \delta)}=\frac{\sqrt{\alpha^{\prime} \tau_{2}}}{2 R} \sum_{k, l \in \mathbf{Z}} \exp \left[-\pi \tau_{2}\left\{\frac{\alpha^{\prime}}{4 R^{2}}(2 l+\delta)^{2}+\frac{4 R^{2}}{\alpha^{\prime}}(k+\epsilon / 2)^{2}\right\}+2 \pi i \tau_{1}(2 l+\delta)(k+\epsilon / 2)\right] .
$$

Then we find that

$$
\begin{aligned}
Z_{1 / R}\left(A_{Y}^{I}\right)=\int \frac{d^{2} \tau}{\tau_{2}} \tau_{2}^{-5}|\eta(\tau)|^{-16} & \left\{\mathcal{Z}_{(0,0)}\left(Z_{L}^{(0,0)}(\tau) Z_{1}^{1}(\tau)^{*^{4}}+Z_{L}^{(0,1)}(\tau) Z_{0}^{1}(\tau)^{*^{4}}\right)\right. \\
& +\mathcal{Z}_{(0,1)}\left(Z_{L}^{(0,0)}(\tau) Z_{1}^{1}(\tau)^{*^{4}}-Z_{L}^{(0,1)}(\tau) Z_{0}^{1}(\tau)^{*^{4}}\right) \\
& +\mathcal{Z}_{(1,0)}\left(Z_{L}^{(1,0)}(\tau) Z_{1}^{0}(\tau)^{*^{4}}+Z_{L}^{(1,1)}(\tau) Z_{0}^{0}(\tau)^{*^{4}}\right) \\
& \left.+\mathcal{Z}_{(1,1)}\left(Z_{L}^{(1,0)}(\tau) Z_{1}^{0}(\tau)^{*^{4}}-Z_{L}^{(1,1)}(\tau) Z_{0}^{0}(\tau)^{*^{4}}\right)\right\}
\end{aligned}
$$

Surprisingly, this partition function can be interpreted as that of an orbifold. The orbifold can be constructed from the supersymmetric heterotic theory by the $\mathbf{Z}_{2}$ twist whose generator is $(-1)^{F_{s}} \gamma_{\delta} \sigma_{1 / 2}$. Here $\sigma_{1 / 2}$ is the half-shift operator [18], and the other two are the same operators as the ones explained in section 4. The sum (5.21) is equal to the length squared of the shift vector $\delta$, and the requirement of the periodicity (5.20) is equivalent to the idenpotency of $\gamma_{\delta}$, i.e. $\gamma_{\delta}^{2}=1$.

Consider the $R \rightarrow 0$ limit. Then $\mathcal{Z}_{(0,1)}$ and $\mathcal{Z}_{(1,1)}$ vanishes, while the other two sums approach the same non-zero value which is independent of $\tau_{2}$. What suvive this limit are the followings.

$$
\begin{aligned}
Z_{1 / R}\left(A_{Y}^{I}\right) \rightarrow \int \frac{d^{2} \tau}{\tau_{2}} \tau_{2}^{-5}|\eta(\tau)|^{-16} & \left\{\left(Z_{L}^{(0,0)}(\tau) Z_{1}^{1}(\tau)^{*^{4}}+Z_{L}^{(0,1)}(\tau) Z_{0}^{1}(\tau)^{*^{4}}\right)\right. \\
+ & \left.\left(Z_{L}^{(1,0)}(\tau) Z_{1}^{0}(\tau)^{*^{4}}+Z_{L}^{(1,1)}(\tau) Z_{0}^{0}(\tau)^{*^{4}}\right)\right\}
\end{aligned}
$$

This is the partition function for a non-supersymmetric heterotic theory compactified on the vanishing $S^{1}$.

Thus we conclude that a supersymmetric heterotic theory on the critical Melvin background with the appropriate Wilson line have the same mass spectrum as that of a 
non-supersymmetric heterotic theory, in the $R \rightarrow 0$ limit. This result can be understood, in the T-dual picture, as evidence of the equivalence between the supersymmetric theory on a non-trivial background and a non-supersymmetric theory, as in the IIA-0A case.

The result for the case with odd values of the sum (5.21) is almost the same. The only defference is the sign in front of $Z_{0}^{0}(\tau)^{* 4}$. This change of sign corresponds to the fact that the eigenvalues of $(-1)^{F_{s}} \gamma_{\delta}$ are a bit peculiar in the twisted sectors, as mentioned in [10].

We have constructed all non-supersymmetric heterotic theories but one in terms of the supersymmetric heterotic theories. The explicit expressions of the partition functions are shown in the appendix. The only exception is the theory whose gauge group is $E_{8}$. It is argued in [10] that to obtain the heterotic $E_{8}$ theory one has to twist the $E_{8} \times E_{8}$ theory by a $\mathbf{Z}_{2}$ involving the outer automorphism of $E_{8} \times E_{8}$ which exchanges the two $E_{8}$ 's. We will discuss this theory in section 8.

\section{Tachyonic instability}

In the IIA-0A case, the tachyonic instability is interpreted as the one due to pair creations of D6-branes. This argument is based on the analysis of the instability of the Melvin background. We will discuss in this section the instabiliity of the non-supersymmetric heterotic theories in terms of the background fields in the supersymmetric theories.

\subsection{Heterotic T-duality}

We have considered the heterotic theories on the Kaluza-Klein Melvin background with a vanishing radius of the $S^{1}$. So it is not appropriate for the argument on the background fields. The ordinary way to improve this situation is to see in the T-dual picture.

Let us recall the T-duality transformation for the heterotic theories. This can be most easily derived from the sigma-model approach [23].

The sigma-model action of the heterotic theories in a general background is written by superfields as follows,

$$
\begin{aligned}
S= & \frac{i}{2 \pi \alpha^{\prime}} \int d^{2} \sigma d \theta\left\{G_{m n}(\Phi)+B_{m n}(\Phi)\right\} \partial_{+} \Phi^{m} D_{-} \Phi^{n} \\
& +\frac{1}{2 \pi} \int d^{2} \sigma d \theta \Psi^{I}\left\{D_{-} \Psi^{I}-i A_{m}^{I J}(\Phi) D_{-} \Phi^{m} \Psi^{J}\right\}
\end{aligned}
$$

where $\theta$ is the Grassmann coordinate, $m, n=0, \cdots, 9$ and $I, J=1, \cdots, 32$. We have defined the real bosonic superfields $\Phi^{m}$ and real fermionic ones $\Psi^{I}$ as follows,

$$
\Phi^{m}=X^{m}+i \sqrt{2 \alpha^{\prime}} \theta \psi_{R}^{m}
$$




$$
\Psi^{I}=\lambda_{L}^{I}+\theta F^{I}
$$

where we have employed the RNS formalism for both left- and right-moving fermions. The covariant derivative $D_{-}$is

$$
D_{-}=\partial_{\theta}-2 i \theta \partial_{-}
$$

After the integration of $\theta$ and integrating out the auxiliary fields $F^{I}$, the action 6.1 becomes

$$
\begin{aligned}
S=\int d^{2} \sigma & {\left[\frac{1}{\pi \alpha^{\prime}}\left\{G_{m n}(X)+B_{m n}(X)\right\} \partial_{+} X^{m} \partial_{-} X^{n}\right.} \\
& +\frac{i}{\pi} G_{m n}(X) \psi_{R}^{m}\left\{\partial_{+} \psi_{R}^{n}+\frac{1}{2}\left(\Gamma_{k l}^{n}(X)+H_{k l}^{n}(X)\right) \partial_{+} X^{k} \psi_{R}^{l}\right\} \\
& \left.+\frac{i}{\pi} \lambda_{L}^{I}\left\{\partial_{-} \lambda^{I}-i A_{m}^{I J}(X) \partial_{-} X^{m} \lambda_{L}^{J}\right\}-i \alpha^{\prime} F_{m n}^{I J}(X) \lambda_{L}^{I} \lambda_{L}^{J} \psi_{R}^{m} \psi_{R}^{n}\right] .
\end{aligned}
$$

The action (5.1) is obtained by substituting the metric of the Melvin background and the constant gauge fields.

Now suppose that the background fields are independent of a coordinate, say, $x^{9}$, or in other words there is an isometry along the 9-direction. Then there exists a dual sigmamodel which is equivalent to the original model. This can be shown by considering the following first order action,

$$
\begin{aligned}
S_{1 s t}=\int d^{2} \sigma & d \theta\left[\frac { i } { 2 \pi \alpha ^ { \prime } } \left(\left\{G_{i j}+B_{i j}\right\} \partial_{+} \Phi^{i} D_{-} \Phi^{j}+i\left\{G_{i 9}+B_{i 9}\right\} \partial_{+} \Phi^{i} V_{-}\right.\right. \\
+ & \left.\left\{G_{9 j}+B_{9 j}\right\} V_{+} D_{-} \Phi^{j}+i G_{99} V_{+} V_{-}\right) \\
+ & \frac{1}{2 \pi}\left(\Psi^{I}\left(D_{-} \Psi^{I}-i A_{i}^{I J} D_{-} \Phi^{i} \Psi^{J}\right)+\Psi^{I} A_{9}^{I J} V_{-} \Psi^{J}\right) \\
+ & \left.\tilde{\Phi}\left(i \partial_{+} V_{-}-D_{-} V_{+}\right)\right]
\end{aligned}
$$

where $i, j=0, \cdots, 8$. We have introduced a bosonic and a fermionic superfields $V_{+}, V_{-}$ respectively, and $\tilde{\Phi}$ is a bosonic Lagrange multiplier superfield.

When the $\tilde{\Phi}$ integration is performed, the superfields $V_{+}, V_{-}$are determined to be

$$
V_{+}=\partial_{+} \Phi^{9}, \quad V_{-}=-i D_{-} \Phi^{9},
$$

for a real bosonic superfield $\Phi^{9}$. Then the action (6.6) becomes the original action (6.1).

On the other hand, when one integrates out $V_{+}, V_{-}$first, which can be done exactly, one obtains a sigma-model with the background fields $\tilde{G}_{\mu \nu}, \tilde{B}_{\mu \nu}, \tilde{A}_{\mu}^{I J}$ such that 23.

$$
\begin{aligned}
& \tilde{G}_{i j}=G_{i j}-\frac{1}{G_{99}}\left(G_{i 9} G_{9 j}+B_{i 9} B_{9 j}\right), \quad \tilde{B}_{i j}=B_{i j}-\frac{1}{G_{99}}\left(G_{i 9} B_{9 j}-G_{j 9} B_{9 i}\right), \\
& \tilde{G}_{i 9}=-\frac{1}{G_{99}} B_{i 9}, \quad \tilde{B}_{i 9}=-\frac{1}{G_{99}} G_{i 9}, \quad \tilde{G}_{99}=\frac{1}{G_{99}} \\
& \tilde{A}_{i}^{I J}=A_{i}^{I J}-\frac{1}{G_{99}}\left(G_{9 i}+B_{9 i}\right) A_{9}^{I J}, \quad \tilde{A}_{9}^{I J}=-\frac{1}{G_{99}} A_{9}^{I J} .
\end{aligned}
$$


(We have set $\alpha^{\prime}=1$.) Note that the dilaton field $\phi$ is also transformed as

$$
\tilde{\phi}=\phi-\frac{1}{2} \log G_{99} .
$$

\subsection{T-dual of Melvin background}

We can now consider the T-dual of the Melvin background. First let us rescale the $y$ coordinate so that its period is $2 \pi$. Then applying the transformation rules (6.8), one obtains

$$
\begin{aligned}
d s_{d u a l}^{2} & =\eta_{\mu \nu} d x^{\mu} d x^{\nu}+d r^{2}+\frac{r^{2}}{1+q^{2} r^{2}} d \theta^{2}+\frac{1}{R^{2}\left(1+q^{2} r^{2}\right)} d y^{2}, \\
\tilde{B}_{\theta y} & =-\frac{q r^{2}}{R\left(1+q^{2} r^{2}\right)}, \\
\tilde{A}_{\theta}^{I J} & =-\frac{q r^{2}}{R^{2}\left(1+q^{2} r^{2}\right)} a^{I J}, \quad \tilde{A}_{y}^{I J}=-\frac{1}{R^{3}\left(1+q^{2} r^{2}\right)} a^{I J},
\end{aligned}
$$

where $a^{I J}=R A_{y}^{I J}$ is independent of both $R$ and $q$. The region $r \ll q^{-1}$ is almost flat and the radius of the $S^{1}$ is large, so this background would be reasonable in this region. An important property of this background is that there exists a nontrivial Yang-Mills field strength.

$$
\begin{aligned}
& \tilde{F}_{78}^{I J}=-\frac{2 q a^{I J}}{R^{2}\left(1+q^{2} r^{2}\right)^{2}} \\
& \tilde{F}_{89}^{I J}=\frac{2 q^{2} a^{I J}}{R^{3}\left(1+q^{2} r^{2}\right)^{2}} x^{8} \\
& \tilde{F}_{97}^{I J}=-\frac{2 q^{2} a^{I J}}{R^{3}\left(1+q^{2} r^{2}\right)^{2}} x^{7}
\end{aligned}
$$

In particular, around the center $r=0$, there is a magnetic field along the 9-direction. This magnetic field would cause, for example, monopole pair creations, and this system would decay into a stable background. This might be the dual picture of the closed string tachyon condensation in the non-supersymmetric heterotic theories. It seems natural to relate the tachyonic instability to the existence of the Yang-Mills magnetic field since the closed string tachyons are, in general, in a nontrivial representation of the gauge group.

Note that for large $r, G_{\theta \theta}$ approaches $q^{-2}$. So the analysis based on the background wolud not be valid when $q$ is large. Thus the discussion given above could not apply to the critical Melvin background, i.e. the case with $q=1 / R$. Nevertheless, we expect that the above-mentioned property still holds at the critical value of $q$.

It is interesting to consider the case in which there is no Wilson line in the original background. Then the gauge field is again absent in the T-dual picture. So there is no 
instability due to the Yang-Mills field strength. In fact, the situation is very close to the IIA-0A case. It is discussed in [17] that the background is stable if there exists a fermion. In Type 0A theory, there is no spacetime fermion and the background is unstable due to the bubble nucleation [17]. On the other hand, in the heterotic theories there are many spacetime fermions, so this may indicate that the critical Melvin background is stable in this case. This is consistent with the fact that the partition function vanishes at $q=1 / R$ if there is no Wilson line, suggesting that the theory becomes supersymmetric.

\section{Strong coupling behavior}

In this section, we will investigate the strong coupling region of the non-supersymmetric heterotic theories. The discussions so far are mainly based on the weakly-coupled string theory, and we have not discussed quantum corrections at all. Because of the absence of the supersymmetry, there is no argument which ensures that the tree-level analysis is enough. There are several arguments for the dual theory of non-supersymmetric theories [18] 24] [25].

Our starting point is the existence of the dual picture shown in the previous section. The advantage to use this fact is that the duality transformations of the supersymmetric theory are available to investigate the dual of a non-supersymmetric theory. So our arguments would be based on a rather firmer footing, even if there is no supersymmetry.

Consider a non-supersymmetric heterotic theory compactified on $S^{1}$ with radius $R$. Denote its coupling constant as $g$. Its strong coupling behaviour would be described by a strongly-coupled supersymmetric heterotic theory on the critical Melvin background, in the $R \rightarrow 0$ limit. The latter theory is either the $S O(32)$ theory or the $E_{8} \times E_{8}$ theory, according to which theory we consider as the former.

(i) The $S O(32)$ theory

What we consider now is the strongly-coupled $S O(32)$ theory on a nontrivial background. Its weak coupling dual is well-known [26] to be Type I theory on the following background.

$$
\begin{aligned}
& G_{I, m n}=e^{-\phi_{I}} G_{h, m n} \\
& \phi_{I}=-\phi_{h} \\
& A_{I, m}=A_{h, m}
\end{aligned}
$$

The subscripts $I, h$ indicate Type I theory and the heterotic theory, respectively. Note that in our case, there is no R-R background in the dual Type I background. The dilaton $\phi_{h}$ is a constant, so that the Type I metric is also the Melvin background. 
Type I theory can be understood as an orientifold of Type IIB theory [27]. So our dual theory is the orientifold of Type IIB theory on the critical Melvin. It can be shown that Type IIB theory on the critical Melvin has the same partition function as that of Type 0B theory in the $R \rightarrow 0$ limit, just as in the IIA-0A case. Therefore the dual theory can be further reinterpreted as an orientifold of Type 0B theory.

Interestingly enough, this orientifold is what was proposed as the dual theory of the $S O(16) \times S O(16)$ heterotic theory 25]. More precisely, in 25] the dual theory is constructed as an orientifold of an interpolating model which relates Type IIB theory to Type OB theory. The partition function of the interpolating model coincides with that of Type IIB theory on the critical Melvin with a finite radius. So the dual theory proposed in [25] is the same as our dual theory. Note that the discussion in 25 is based on deformations of the supersymmetric theories, and it is not obvious whether the Type I-heterotic duality can be applied in this case, as mentioned by the authers. Since now we have related the non-supersymmetric theories to the supersymmetric theories on some background, there would be no obstruction to apply the duality relation.

The pieces of evidence for the Type 0B orientifold to be the dual of the non- supersymmetric $S O(16) \times S O(16)$ theory are the followings [25].

1. The massless spectrum is the same.

2. It is argued that fluctuations on the D1-brane in the Type 0B orientifold corresponds to the worldsheet fields in non-supersymmetric theory, under a few assumptions.

These are nice properties for them to be a dual pair. It will be interesting to confirm the discussions in 25] from our point of view based on the supersymmetric theory.

Since in the Type 0B orientifold picture the radius of the $y$-direction is still small, we have to take the T-dual transformation along the $y$-direction. Then we obtain Type 0A theory compactified on $S^{1} / \mathbf{Z}_{2}$. It would be natural to expect that the Type $0 \mathrm{~A}$ orbifold decays into a Type IIA orbifold, just like the decay in the IIA-0A case in ten dimensions. (Note that the decay process would be different from the IIA-0A case, in particular, in the Type 0A orbifold which is related to the $S O(16) \times S O(16)$ heterotic theory, since the orbifold does not contain any tachyon.) It is well-known that the Type IIA orbifold is the dual of a supersymmetric heterotic theory (with an appropriate Wilson line). Therefore we conclude that the picture of the decay of the non-supersymmetric theories obtained from the perturbative analysis still hold in the strong coupling region.

Let us determine the coupling constant $g_{0 A}$ and the length $R_{0 A}$ of the segment $S^{1} / \mathbf{Z}_{2}$ in the Type $0 \mathrm{~A}$ orbifold when $g$ is large and $R$ is small in the corresponding nonsupersymmertic theory. Applying the succesive duality transformations, one finds

$$
g_{0 A} \sim g^{-\frac{1}{2}} R^{-1}, \quad R_{0 A} \sim g^{\frac{1}{2}} R^{-1},
$$

up to numerical factors.

For large but finite $g$, the segmant is large but the coupling constant also becomes large. This would not so problematic since the arguments on the IIA-0A case is not restricted in the perturbative region. One can obtain, if necessary, a weakly-coupled 
Type 0A orbifold as the dual theory, by taking the following limit.

$$
g \rightarrow \infty, \quad R \rightarrow 0, \quad g R^{2}: \text { large but finite }
$$

(ii) The $E_{8} \times E_{8}$ theory

The dual of the strongly-coupled $E_{8} \times E_{8}$ theory on the critical Melvin is rather easy to obtain. It is M-theory on $\mathbf{R}^{9} \times S^{1} \times S^{1} / \mathbf{Z}_{2}$, with the antiperiodic boundary condition for fermions along the $S^{1}$. After reducing M-theory along the $S^{1} / \mathbf{Z}_{2}$, this corresponds to the non-supersymmetric heterotic theory in the $R \rightarrow 0$ limit, where $R$ is the radius of the $S^{1}$. Then, exchanging the roles of the $S^{1}$ and the $S^{1} / \mathbf{Z}_{2}$, and reducing the theory along the $S^{1}$ direction, we obtain a weakly-coupled Type 0 A theory on the $S^{1} / \mathbf{Z}_{2}$. This is because M-theory compactified on such a "twisted circle" corresponds to Type 0A theory [18], which is one of the main point of the IIA-0A duality. Thus we again find the Type $0 \mathrm{~A}$ orbifold as the dual theory of the non-supersymmetric heterotic theories. So the nonsupersymmetric theories, which are related to the $E_{8} \times E_{8}$ theory, are expected to decay into the supersymmetric theroy even in the strong coupling region.

\section{Discussions}

We have constructed the non-supersymmetric heterotic theories as the supersymmetric heterotic theories on the critical Melvin background with the Wilson lines. However, we have not discussed such a construction of the $E_{8}$ theory. This theory is rather exceptional; for example, the rank of its gauge group is eight, while all the other theories have the gauge group of the rank sixteen. Moreover, to construct the $E_{8}$ theory via the twisting procedure from the $E_{8} \times E_{8}$ theory, the $\mathbf{Z}_{2}$ group should consist of the outer automorphism of $E_{8} \times E_{8}$ which exchanges the two $E_{8}$ 's. So this theory does not seem to be constructed as a theory on the critical Melvin. One possible supersymmetric theory which can be related to this theory is the CHL theory [28]. The CHL theory can have the gauge group of the rank eight, and it can be constructed by twisting the $E_{8} \times E_{8}$ theory by a $\mathbf{Z}_{2}$ which consists

of the outer automorphism discussed above. Since the CHL theory is supersymmetric, it would be natural to expect that the $E_{8}$ theory decays into this theory.

The non-supersymmetric theories have been related to the critical Melvin background in the supersymmetric theories. This background has nontrivial field strength, either in the lower-dimensional or T-dual point of view. Such configurations have nonzero energies, compared with the supersymmetric vacuum. So one is led to a conjecture that the "vacuum energies" of the nonsupersymmetric theories are, in general, positive and their values are given by the energies of the field strength in the dual supersymmetric theories. This might have something to do with the fact that the one loop cosmological constant of the $S O(16) \times S O(16)$ theory is positive 10 . 
In section 7, we have discussed the strong coupling behavior of the non-supersymmetric theories. This kind of analysis is in general very difficult because of the absence of supersymmetry. In our case, it can be done since the non-supersymmetric theories are related to the non-supersymmetric backgrounds in the supersymmetric theories, and the duality transformations for the background fields are well-known. This seems to be a very suitable strategy to discuss the duality between non-supersymmetric theories. It would be very interesting if this strategy can apply to some other non-supersymmetric theories.

One of our motivations to study the Melvin background in string theory is to understand the mechanism of the stabilization of closed string tachyons. In [5] [6], the tachyon condensation is related to pair creations of D6-branes in Type IIA picture. However, the corresponding picture in Type 0A theory is not yet clarified. The bubble nucleation in [17] pushes our world to infinity, and there remains nothing. It would be very important to understand this phenomenon in terms of Type 0A theory language, and clarify the mechanism of the closed string tachyon condensation.

In the heterotic theories, the situation is more complicated. Since most of the theories have tachyons in a nontrivial representation of their gauge groups, the condensation of the tachyons would break the gauge symmetry. Therefore, even if the endpoint of the condensation is a supersymmetric theory, the pattern of the Wilson line would be defferent from the original one. This might suggest that the marginal deformation of the worldsheet CFT, which corresponds to the change in $q$ alone, would have nothing to do with the tachyon condensation.

\section{Acknowledgements}

I would like to thank S. Iso, H. Itoyama and T. Matsuo for valuable discussions. 


\section{A Explicit expressions of partition functions}

In this appendix, we will show explicitly the partition functions $Z_{1 / R}\left(A_{y}^{I}\right)$ discussed in section 5. First we have to choose the appropriate Wilson lines [10]. We assumed the periodicity of the left-moving contribution (5.20). This means that the sixteen dimensional vector

$$
\delta=\left(a^{1}, \cdots, a^{16}\right)
$$

has the property that $2 \delta$ lies in the momentum lattice, for a suitable choice of the basis vectors. Such vectors are classified in [29] and the followings are the representatives of the equivalence classes. For the $S O(32)$ theory,

$$
\delta=\left(1,0^{15}\right),\left(\left(\frac{1}{2}\right)^{4}, 0^{12}\right),\left(\left(\frac{1}{4}\right)^{16}\right),\left(\left(\frac{1}{2}\right)^{8}, 0^{8}\right)
$$

and for the $E_{8} \times E_{8}$ theory,

$$
\delta=\left(1,0^{7} ; 0^{8}\right),\left(\left(\frac{1}{2}\right)^{2}, 0^{6} ;\left(\frac{1}{2}\right)^{2}, 0^{6}\right),\left(1,0^{7} ; 1,0^{7}\right) .
$$

Below we denote the partition functions for various sectors as

$$
\begin{aligned}
\chi_{N S \pm}^{(n)} & =\frac{1}{2}\left(Z_{0}^{0}(\tau)^{n} \pm Z_{1}^{0}(\tau)^{n}\right), \\
\chi_{R}^{(n)} & =\frac{1}{2} Z_{0}^{1}(\tau)^{n},
\end{aligned}
$$

for the left-movers and

$$
\begin{aligned}
\bar{\chi}_{N S \pm} & =\frac{1}{2}\left(Z_{0}^{0}(\tau)^{4} \mp Z_{1}^{0}(\tau)^{4}\right)^{*} \\
\bar{\chi}_{R} & =\frac{1}{2} Z_{0}^{1}(\tau)^{4^{*}}
\end{aligned}
$$

for the right-movers.

\section{A.1 The $S O(32)$ theory}

(i) $\delta=\left(1,0^{15}\right)$

In this case, the partition function $Z_{L}^{(m, n)}(\tau)$ for the left-movers are

$$
Z_{L}^{(m, n)}(\tau)=Z_{0}^{0}(\tau)^{16}+e^{-\pi i m} Z_{1}^{0}(\tau)^{16}+e^{\pi i n} Z_{0}^{1}(\tau)^{16} .
$$

Then the full partition function is

$$
Z_{1 / R}\left(A_{y}^{I}\right)=\int \frac{d^{2} \tau}{\tau_{2}} \tau_{2}^{-5}|\eta(\tau)|^{-16} Z_{F}
$$


where

$$
\begin{aligned}
& Z_{F}=4\left\{\mathcal{Z}_{(0,0)}\left(\chi_{N S+}^{(16)} \bar{\chi}_{N S+}-\chi_{R}^{(16)} \bar{\chi}_{R}\right)+\mathcal{Z}_{(0,1)}\left(\chi_{R}^{(16)} \bar{\chi}_{N S+}-\chi_{N S+}^{(16)} \bar{\chi}_{R}\right)\right. \\
& \left.+\mathcal{Z}_{(1,0)}\left(\chi_{N S-}^{(16)} \bar{\chi}_{N S-}-\chi_{R}^{(16)} \bar{\chi}_{R}\right)+\mathcal{Z}_{(0,1)}\left(\chi_{R}^{(16)} \bar{\chi}_{N S-}-\chi_{N S-}^{(16)} \bar{\chi}_{R}\right)\right\} .
\end{aligned}
$$

In the $R \rightarrow 0$ limit,

$$
Z_{F} \propto \chi_{N S+}^{(16)} \bar{\chi}_{N S+}+\chi_{N S-}^{(16)} \bar{\chi}_{N S-}-2 \chi_{R}^{(16)} \bar{\chi}_{R}
$$

So $Z_{1 / R}\left(A_{y}^{I}\right)$ coincides exactly with the partition function for the non-supersymmetric $S O(32)$ theory in this limit.

(ii) $\delta=\left(\left(\frac{1}{2}\right)^{4}, 0^{12}\right)$

In this case, from the expression

$$
Z_{L}^{(m, n)}(\tau)=Z_{n}^{m}(\tau)^{4} Z_{0}^{0}(\tau)^{12}+Z_{n+1}^{m}(\tau)^{4} Z_{1}^{0}(\tau)^{12}+Z_{n}^{m+1}(\tau)^{4} Z_{0}^{1}(\tau)^{12}
$$

one can find

$$
\begin{aligned}
Z_{F}=4[ & \mathcal{Z}_{(0,0)}\left\{\left(\chi_{N S+}^{(4)} \chi_{N S+}^{(12)}+\chi_{R}^{(4)} \chi_{R}^{(12)}\right) \bar{\chi}_{N S+}-\left(\chi_{N S-}^{(4)} \chi_{N S-}^{(12)}+\chi_{R}^{(4)} \chi_{R}^{(12)}\right) \bar{\chi}_{R}\right\} \\
& +\mathcal{Z}_{(0,1)}\left\{\left(\chi_{N S-}^{(4)} \chi_{N S-}^{(12)}+\chi_{R}^{(4)} \chi_{R}^{(12)}\right) \bar{\chi}_{N S+}-\left(\chi_{N S+}^{(4)} \chi_{N S+}^{(12)}+\chi_{R}^{(4)} \chi_{R}^{(12)}\right) \bar{\chi}_{R}\right\} \\
& +\mathcal{Z}_{(1,0)}\left\{\left(\chi_{R}^{(4)} \chi_{N S+}^{(12)}+\chi_{N S+}^{(4)} \chi_{R}^{(12)}\right) \bar{\chi}_{N S-}-\left(\chi_{R}^{(4)} \chi_{N S-}^{(12)}+\chi_{N S-}^{(4)} \chi_{R}^{(12)}\right) \bar{\chi}_{R}\right\} \\
& \left.+\mathcal{Z}_{(1,1)}\left\{\left(\chi_{R}^{(4)} \chi_{N S-}^{(12)}+\chi_{N S-}^{(4)} \chi_{R}^{(12)}\right) \bar{\chi}_{N S-}-\left(\chi_{R}^{(4)} \chi_{N S+}^{(12)}+\chi_{N S+}^{(4)} \chi_{R}^{(12)}\right) \bar{\chi}_{R}\right\}\right] .
\end{aligned}
$$

In the $R \rightarrow 0$ limit,

$$
\begin{aligned}
Z_{F} \propto & \left(\chi_{N S+}^{(4)} \chi_{N S+}^{(12)}+\chi_{R}^{(4)} \chi_{R}^{(12)}\right) \bar{\chi}_{N S+}-\left(\chi_{N S-}^{(4)} \chi_{N S-}^{(12)}+\chi_{R}^{(4)} \chi_{R}^{(12)}\right) \bar{\chi}_{R} \\
& +\left(\chi_{R}^{(4)} \chi_{N S+}^{(12)}+\chi_{N S+}^{(4)} \chi_{R}^{(12)}\right) \bar{\chi}_{N S-}-\left(\chi_{R}^{(4)} \chi_{N S-}^{(12)}+\chi_{N S-}^{(4)} \chi_{R}^{(12)}\right) \bar{\chi}_{R}
\end{aligned}
$$

The corresponding shift operator $\gamma_{\delta}$ can be represented as

$$
\begin{aligned}
\gamma_{\delta} & =e^{\pi i\left(J_{12}+J_{34}+J_{56}+J_{78}\right)} \\
& =(-1)^{F^{(4)}}
\end{aligned}
$$

where $F^{(4)}$ counts the number of $\lambda_{L}^{1}, \cdots, \lambda_{L}^{4}$. Therefore one can see that the partition function in the $R \rightarrow 0$ limit coincides with that of the supersymmetric $S O(32)$ theory twisted by $(-1)^{F_{s}} \gamma_{\delta}$, which is the $S O(8) \times S O(24)$ theory.

$$
\text { (iii) } \delta=\left(\left(\frac{1}{4}\right)^{16}\right)
$$


The expression for the left-moving contribution is

$$
Z_{L}^{(m, n)}(\tau)=Z_{n / 2}^{m / 2}(\tau)^{16}+Z_{1+n / 2}^{m / 2}(\tau)^{16}+Z_{n / 2}^{1+m / 2}(\tau)^{16}+Z_{1+n / 2}^{1+m / 2}(\tau)^{16} .
$$

The periodicity (5.20) is accomplished by permuting the terms in the RHS.

The partition function is given by

$$
\begin{aligned}
Z_{F}=4[ & \mathcal{Z}_{(0,0)}\left\{\left(\chi_{N S+}^{+}+\chi_{R+}^{+}\right) \bar{\chi}_{N S+}-\left(\chi_{N S+}^{-}+\chi_{R+}^{-}\right) \bar{\chi}_{R}\right\} \\
& +\mathcal{Z}_{(0,1)}\left\{\left(\chi_{N S+}^{-}+\chi_{R+}^{-}\right) \bar{\chi}_{N S+}-\left(\chi_{N S+}^{+}+\chi_{R+}^{+}\right) \bar{\chi}_{R}\right\} \\
& +\mathcal{Z}_{(1,0)}\left\{\left(\tilde{\chi}_{N S+}^{+}+\tilde{\chi}_{R+}^{+}\right) \bar{\chi}_{N S-}-\left(\tilde{\chi}_{N S+}^{-}+\tilde{\chi}_{R+}^{-}\right) \bar{\chi}_{R}\right\} \\
& \left.+\mathcal{Z}_{(1,1)}\left\{\left(\tilde{\chi}_{N S+}^{-}+\tilde{\chi}_{R+}^{-}\right) \bar{\chi}_{N S-}-\left(\tilde{\chi}_{N S+}^{+}+\tilde{\chi}_{R+}^{+}\right) \bar{\chi}_{R}\right\}\right]
\end{aligned}
$$

where

$$
\begin{aligned}
\chi_{N S+}^{ \pm} & =\frac{1}{4}\left(Z_{0}^{0}(\tau)^{16}+Z_{1}^{0}(\tau)^{16} \pm\left(Z_{\frac{1}{2}}^{0}(\tau)^{16}+Z_{1+\frac{1}{2}}^{0}(\tau)^{16}\right)\right), \\
\chi_{R+}^{ \pm} & =\frac{1}{4}\left(Z_{0}^{1}(\tau)^{16}+Z_{1}^{1}(\tau)^{16} \pm\left(Z_{\frac{1}{2}}^{1}(\tau)^{16}+Z_{1+\frac{1}{2}}^{1}(\tau)^{16}\right)\right), \\
\tilde{\chi}_{N S+}^{ \pm} & =\frac{1}{4}\left(Z_{0}^{\frac{1}{2}}(\tau)^{16}+Z_{1}^{\frac{1}{2}}(\tau)^{16} \pm\left(Z_{\frac{1}{2}}^{\frac{1}{2}}(\tau)^{16}+Z_{1+\frac{1}{2}}^{\frac{1}{2}}(\tau)^{16}\right)\right), \\
\tilde{\chi}_{R+}^{ \pm} & =\frac{1}{4}\left(Z_{0}^{1+\frac{1}{2}}(\tau)^{16}+Z_{1}^{1+\frac{1}{2}}(\tau)^{16} \pm\left(Z_{\frac{1}{2}}^{1+\frac{1}{2}}(\tau)^{16}+Z_{1+\frac{1}{2}}^{1+\frac{1}{2}}(\tau)^{16}\right)\right) .
\end{aligned}
$$

Note that $\chi_{N S+(R+)}^{ \pm}$are the projected partition functions

$$
\chi_{N S+(R+)}^{ \pm}=\operatorname{Tr}_{N S(R)} \frac{1+(-1)^{F}}{2} \frac{1 \pm \gamma_{\delta}}{2} q^{H},
$$

where $\gamma_{\delta}$ acts as $e^{\frac{\pi}{2} i}$ on all $\lambda_{L}^{I}$ 's. The other two functions $\tilde{\chi}_{N S+(R+)}^{ \pm}$are the projected partition functions for the twisted sectors.

In the $R \rightarrow 0$ limit,

$$
\begin{aligned}
Z_{F} \propto & \left(\chi_{N S+}^{+}+\chi_{R+}^{+}\right) \bar{\chi}_{N S+}-\left(\chi_{N S+}^{-}+\chi_{R+}^{-}\right) \bar{\chi}_{R} \\
+ & \left(\tilde{\chi}_{N S+}^{+}+\tilde{\chi}_{R+}^{+}\right) \bar{\chi}_{N S-}-\left(\tilde{\chi}_{N S+}^{-}+\tilde{\chi}_{R+}^{-}\right) \bar{\chi}_{R} .
\end{aligned}
$$

This is the fermion part of the partition function for the non-supersymmetric $U(16)$ theory.

$$
\text { (iv) } \delta=\left(\left(\frac{1}{2}\right)^{8}, 0^{8}\right)
$$

The calculation is similar to the one in the case (ii) above, except for the extra signs in the twisted sectors. The partition function is then given by

$$
Z_{F}=4\left[\mathcal{Z}_{(0,0)}\left\{\left(\chi_{N S+}^{(8)} \chi_{N S+}^{(8)}+\chi_{R}^{(8)} \chi_{R}^{(8)}\right) \bar{\chi}_{N S+}-\left(\chi_{N S-}^{(8)} \chi_{N S-}^{(8)}+\chi_{R}^{(8)} \chi_{R}^{(8)}\right) \bar{\chi}_{R}\right\}\right.
$$




$$
\begin{aligned}
& +\mathcal{Z}_{(0,1)}\left\{\left(\chi_{N S-}^{(8)} \chi_{N S-}^{(8)}+\chi_{R}^{(8)} \chi_{R}^{(8)}\right) \bar{\chi}_{N S+}-\left(\chi_{N S+}^{(8)} \chi_{N S+}^{(8)}+\chi_{R}^{(8)} \chi_{R}^{(8)}\right) \bar{\chi}_{R}\right\} \\
& +\mathcal{Z}_{(1,0)}\left\{2 \chi_{R}^{(8)} \chi_{N S-}^{(8)} \bar{\chi}_{N S-}-2 \chi_{R}^{(8)} \chi_{N S+}^{(8)} \bar{\chi}_{R}\right\} \\
& \left.+\mathcal{Z}_{(1,1)}\left\{2 \chi_{R}^{(8)} \chi_{N S+}^{(8)} \bar{\chi}_{N S-}-2 \chi_{R}^{(8)} \chi_{N S-}^{(8)} \bar{\chi}_{R}\right\}\right] .
\end{aligned}
$$

In the $R \rightarrow 0$ limit,

$$
\begin{aligned}
Z_{F} \propto & \left(\chi_{N S+}^{(8)} \chi_{N S+}^{(8)}+\chi_{R}^{(8)} \chi_{R}^{(8)}\right) \bar{\chi}_{N S+}-\left(\chi_{N S-}^{(8)} \chi_{N S-}^{(8)}+\chi_{R}^{(8)} \chi_{R}^{(8)}\right) \bar{\chi}_{R} \\
& +2 \chi_{R}^{(8)} \chi_{N S-}^{(8)} \bar{\chi}_{N S-}-2 \chi_{R}^{(8)} \chi_{N S+}^{(8)} \bar{\chi}_{R} .
\end{aligned}
$$

This coincides with the fermion part of the partition function for the non-supersymmetric $S O(16) \times S O(16)$ theory.

\section{A.2 The $E_{8} \times E_{8}$ theory}

(v) $\delta=\left(1,0^{7} ; 0^{8}\right)$

From the similar calculation to the one in the case (i),

$$
\begin{aligned}
& Z_{F}=8\left(\chi_{N S+}^{(8)}+\chi_{R}^{(8)}\right)\left\{\mathcal{Z}_{(0,0)}\left(\chi_{N S+}^{(8)} \bar{\chi}_{N S+}-\chi_{R}^{(8)} \bar{\chi}_{R}\right)+\mathcal{Z}_{(0,1)}\left(\chi_{R}^{(8)} \bar{\chi}_{N S+}-\chi_{N S+}^{(8)} \bar{\chi}_{R}\right)\right. \\
& \left.+\mathcal{Z}_{(1,0)}\left(\chi_{N S-}^{(8)} \bar{\chi}_{N S-}-\chi_{R}^{(8)} \bar{\chi}_{R}\right)+\mathcal{Z}_{(1,1)}\left(\chi_{R}^{(8)} \bar{\chi}_{N S-}-\chi_{N S-}^{(8)} \bar{\chi}_{R}\right)\right\} .
\end{aligned}
$$

The fermion part of the partition function for the non-supersymmetric $E_{8} \times S O(16)$ theory is obtained in the $R \rightarrow 0$ limit.

$$
Z_{F} \propto\left(\chi_{N S+}^{(8)}+\chi_{R}^{(8)}\right)\left(\chi_{N S+}^{(8)} \bar{\chi}_{N S+}+\chi_{N S-}^{(8)} \bar{\chi}_{N S-}-2 \chi_{R}^{(8)} \bar{\chi}_{R}\right)
$$

(vi) $\delta=\left(\left(\frac{1}{2}\right)^{2}, 0^{6} ;\left(\frac{1}{2}\right)^{2}, 0^{6}\right)$

The partition function is a bit complicated in this case.

$$
\begin{aligned}
& Z_{F}=8\left(\mathcal { Z } _ { ( 0 , 0 ) } \left[\left\{\left(\chi_{N S+}^{(2)} \chi_{N S+}^{(6)}+\chi_{R}^{(2)} \chi_{R}^{(6)}\right)^{2}+\left(\chi_{N S-}^{(2)} \chi_{N S-}^{(6)}+\chi_{R}^{(2)} \chi_{R}^{(6)}\right)^{2}\right\} \bar{\chi}_{N S+}\right.\right. \\
&\left.-2\left(\chi_{N S+}^{(2)} \chi_{N S+}^{(6)}+\chi_{R}^{(2)} \chi_{R}^{(6)}\right)\left(\chi_{N S-}^{(2)} \chi_{N S-}^{(6)}+\chi_{R}^{(2)} \chi_{R}^{(6)}\right) \bar{\chi}_{R}\right] \\
&+\mathcal{Z}_{(0,1)}\left[2\left(\chi_{N S+}^{(2)} \chi_{N S+}^{(6)}+\chi_{R}^{(2)} \chi_{R}^{(6)}\right)\left(\chi_{N S-}^{(2)} \chi_{N S-}^{(6)}+\chi_{R}^{(2)} \chi_{R}^{(6)}\right) \bar{\chi}_{N S+}\right. \\
&\left.-\left\{\left(\chi_{N S+}^{(2)} \chi_{N S+}^{(6)}+\chi_{R}^{(2)} \chi_{R}^{(6)}\right)^{2}+\left(\chi_{N S-}^{(2)} \chi_{N S-}^{(6)}+\chi_{R}^{(2)} \chi_{R}^{(6)}\right)^{2}\right\} \bar{\chi}_{R}\right] \\
&+\mathcal{Z}_{(1,0)}\left[\left\{\left(\chi_{R}^{(2)} \chi_{N S+}^{(6)}+\chi_{N S-}^{(2)} \chi_{R}^{(6)}\right)^{2}+\left(\chi_{N S+}^{(2)} \chi_{R}^{(6)}+\chi_{R}^{(2)} \chi_{N S-}^{(6)}\right)^{2}\right\} \bar{\chi}_{N S-}\right. \\
&-\left.-2\left(\chi_{R}^{(2)} \chi_{N S+}^{(6)}+\chi_{N S-}^{(2)} \chi_{R}^{(6)}\right)\left(\chi_{N S+}^{(2)} \chi_{R}^{(6)}+\chi_{R}^{(2)} \chi_{N S-}^{(6)}\right) \bar{\chi}_{R}\right]
\end{aligned}
$$




$$
\begin{aligned}
+\mathcal{Z}_{(1,1)}[ & 2\left(\chi_{R}^{(2)} \chi_{N S+}^{(6)}+\chi_{N S-}^{(2)} \chi_{R}^{(6)}\right)\left(\chi_{N S+}^{(2)} \chi_{R}^{(6)}+\chi_{R}^{(2)} \chi_{N S-}^{(6)}\right) \bar{\chi}_{N S-} \\
& \left.\left.-\left\{\left(\chi_{R}^{(2)} \chi_{N S+}^{(6)}+\chi_{N S-}^{(2)} \chi_{R}^{(6)}\right)^{2}+\left(\chi_{N S+}^{(2)} \chi_{R}^{(6)}+\chi_{R}^{(2)} \chi_{N S-}^{(6)}\right)^{2}\right\} \bar{\chi}_{R}\right]\right)
\end{aligned}
$$

In the $R \rightarrow 0$ limit,

$$
\begin{aligned}
Z_{F} \propto & \left\{\left(\chi_{N S+}^{(2)} \chi_{N S+}^{(6)}+\chi_{R}^{(2)} \chi_{R}^{(6)}\right)^{2}+\left(\chi_{N S-}^{(2)} \chi_{N S-}^{(6)}+\chi_{R}^{(2)} \chi_{R}^{(6)}\right)^{2}\right\} \bar{\chi}_{N S+} \\
- & 2\left(\chi_{N S+}^{(2)} \chi_{N S+}^{(6)}+\chi_{R}^{(2)} \chi_{R}^{(6)}\right)\left(\chi_{N S-}^{(2)} \chi_{N S-}^{(6)}+\chi_{R}^{(2)} \chi_{R}^{(6)}\right) \bar{\chi}_{R} \\
+ & \left\{\left(\chi_{R}^{(2)} \chi_{N S+}^{(6)}+\chi_{N S-}^{(2)} \chi_{R}^{(6)}\right)^{2}+\left(\chi_{N S+}^{(2)} \chi_{R}^{(6)}+\chi_{R}^{(2)} \chi_{N S-}^{(6)}\right)^{2}\right\} \bar{\chi}_{N S+} \\
- & 2\left(\chi_{R}^{(2)} \chi_{N S+}^{(6)}+\chi_{N S-}^{(2)} \chi_{R}^{(6)}\right)\left(\chi_{N S+}^{(2)} \chi_{R}^{(6)}+\chi_{R}^{(2)} \chi_{N S-}^{(6)}\right) \bar{\chi}_{R} .
\end{aligned}
$$

This is the fermion part of the partition function for the non-supersymmetric $\left(E_{7} \times S U(2)\right)^{2}$ theory. Note that $\gamma_{\delta}$ can be divided as $\gamma_{1} \gamma_{2}$, where

$$
\gamma_{1,2}=(-1)^{\epsilon}(-1)^{F_{1,2}^{(2)}} .
$$

The operator $F_{1}^{(2)}$ counts the number of the $\lambda_{L}^{1,2}$ and the operator $F_{2}^{(2)}$ the number of the $\lambda_{L}^{9,10}$, and

$$
\epsilon= \begin{cases}0, & \text { for NS-sector } \\ 1, & \text { for R-sector. }\end{cases}
$$

(vii) $\delta=\left(1,0^{7} ; 1,0^{7}\right)$

One can obtain the following.

$$
\begin{aligned}
Z_{F}=8[ & \mathcal{Z}_{(0,0)}\left\{\left(\chi_{N S+}^{(8)} \chi_{N S+}^{(8)}+\chi_{R}^{(8)} \chi_{R}^{(8)}\right) \bar{\chi}_{N S+}-2 \chi_{N S+}^{(8)} \chi_{R}^{(8)} \bar{\chi}_{R}\right\} \\
+ & \mathcal{Z}_{(0,1)}\left\{2 \chi_{N S+}^{(8)} \chi_{R}^{(8)} \bar{\chi}_{N S+}-\left(\chi_{N S+}^{(8)} \chi_{N S+}^{(8)}+\chi_{R}^{(8)} \chi_{R}^{(8)}\right) \bar{\chi}_{R}\right\} \\
+ & \mathcal{Z}_{(1,0)}\left\{2 \chi_{N S-}^{(8)} \chi_{R}^{(8)} \bar{\chi}_{N S-}-\left(\chi_{N S-}^{(8)} \chi_{N S-}^{(8)}+\chi_{R}^{(8)} \chi_{R}^{(8)}\right) \bar{\chi}_{R}\right\} \\
& \left.+\mathcal{Z}_{(1,1)}\left\{\left(\chi_{N S-}^{(8)} \chi_{N S-}^{(8)}+\chi_{R}^{(8)} \chi_{R}^{(8)}\right) \bar{\chi}_{N S-}-2 \chi_{N S-}^{(8)} \chi_{R}^{(8)} \bar{\chi}_{R}\right\}\right],
\end{aligned}
$$

and, in the $R \rightarrow 0$ limit,

$$
\begin{aligned}
Z_{F} \propto & \left(\chi_{N S+}^{(8)} \chi_{N S+}^{(8)}+\chi_{R}^{(8)} \chi_{R}^{(8)}\right) \bar{\chi}_{N S+}-2 \chi_{N S+}^{(8)} \chi_{R}^{(8)} \bar{\chi}_{R} \\
& +2 \chi_{N S-}^{(8)} \chi_{R}^{(8)} \bar{\chi}_{N S-}-\left(\chi_{N S-}^{(8)} \chi_{N S-}^{(8)}+\chi_{R}^{(8)} \chi_{R}^{(8)}\right) \bar{\chi}_{R} .
\end{aligned}
$$

The last expression is the same as (A.25), although the expressions before taking the limit are different from each other. 


\section{References}

[1] T.Banks, W.Fischler, S.H.Shenker, L.Susskind, M Theory As A Matrix Model: A Conjecture, Phys. Rev. D55 (1997) 5112, hep-th/9610043;

N.Ishibashi, H.Kawai, Y.Kitazawa, A.Tsuchiya, A Large-N Reduced Model as Superstring, Nucl. Phys. B498 (1997) 467, hep-th/9612115.

[2] For a review, see e.g. A.Sen, Non-BPS States and Branes in String Theory, hepth/9904207.

[3] E.Witten, Non-commutative Geometry and String Field Theory, Nucl. Phys. B268 (1986) 253.

[4] L.Rastelli, A.Sen, B.Zwiebach, String Field Theory Around the Tachyon Vacuum, hep-th/0012251; Classical Solutions in String Field Theory Around the Tachyon Vacuum, hep-th/0102112; Half-strings, Projectors, and Multiple D-branes in Vacuum String Field Theory, hep-th/0105058,

I.Ellwood, W.Taylor, Open String Field Theory Without Open Strings, hepth/0103085;

D.J.Gross, W.Taylor, Split String Field Theory I \& II, hep-th/0105059, 0106036.

T.Kawano, K.Okuyama, Open String Fields As Matrices, hep-th/0105129.

[5] M.S.Costa, M.Gutperle, The Kaluza-Klein Melvin Solution in M-theory, JHEP 0103 (2001) 027, hep-th/0012072.

[6] M.Gutperle, A.Strominger, Fluxbranes in String Theory, JHEP 0106 (2001) 035, hep-th/0104136.

[7] J.G.Russo, A.A.Tseytlin, Magnetic Backgrounds and Tachyonic Instabilities in Closed Superstring Theory and M-theory, hep-th/0104238.

[8] M.A.Melvin, Pure Magnetic and Electric Geons, Phys. Lett. B8 (1964) 65.

[9] T.Suyama, Closed String Tachyons in Non-supersymmetric Heterotic Theories, hepth/0106079.

[10] L.J.Dixon, J.A.Harvey, String Theories in Ten Dimensions Without Spacetime Supersymmetry, Nucl. Phys. B274 (1986) 93.

[11] L.Alvarez-Gaume, P.Ginsparg, G.Moore, C.Vafa, An $O(16) \times O(16)$ Heterotic String, Phys. Lett. B171 (1986) 155;

N.Seiberg, E.Witten, Spin Structures in String Theory, Nucl. Phys. B276 (1986) 272.

[12] H.Kawai, D.C.Lewellen, S.-H.H.Tye, Classification of Closed-fermionic-string Models, Phys. Rev. D34 (1986) 3794. 
[13] P.M.Saffin, Gravitating Fluxbranes, Phys.Rev. D64 (2001) 024014, gr-qc/0104014;

M.S.Costa, C.A.R.Herdeiro, L.Cornalba, Flux-branes and the Dielectric Effect in String Theory, hep-th/0105023.

[14] L.Motl, Melvin Matrix Models, hep-th/0107002.

[15] F.Dowker, J.P.Gauntlett, D.A.Kastor, J.Traschen, Pair Creation of Dilaton Black Holes, Phys. Rev. D49 (1994) 2909, hep-th/9309075;

F.Dowker, J.P.Gauntlett, S.B.Giddings, G.T.Horowitz, On Pair Creation of Extremal Black Holes and Kaluza-Klein Monopoles, Phys. Rev. D50 (1994) 2662, hepth/9312172; The Decay of Magnetic Fields in Kaluza-Klein Theory, Phys. Rev D52 (1995) 6929, hep-th/9507143; Nucleation of P-Branes and Fundamental Strings, Phys. Rev. D53 (1996) 7115, hep-th/9512154.

[16] R.C.Myers, M.J.Perry, Black Holes in Higher Dimensional Space-times, Ann. Phys. 172 (1986) 304.

[17] E.Witten, Instability of the Kaluza-Klein Vacuum, Nucl. Phys. B195 (1982) 481.

[18] O.Bergman, M.R.Gaberdiel, Dualities of Type 0 Strings, JHEP 9907 (1999) 022, hep-th/9906055.

[19] A.A.Tseytlin, Closed Superstrings in Magnetic Field: Instabilities and Supersymmetry Breaking, Nucl. Phys. Proc. Suppl. 49 (1996) 338, hep-th/9510041;

J.G.Russo, A.A.Tseytlin, Magnetic Flux Tube Models in Superstring Theory, Nucl. Phys. B461 (1996) 131, hep-th/9508068.

[20] G.T.Horowitz, A.A.Tseytlin, A New Class of Exact Solutions in String Theory, Phys. Rev. D51 (1995) 2896, hep-th/9409021;

J.G.Russo, A.A.Tseytlin, Constant Magnetic Field in Closed String Theory: An Exactly Solvable Model, Nucl. Phys. B448 (1995) 293, hep-th/9411099.

[21] L.Alvarez-Gaume, G.Moore, C.Vafa, Theta Functions, Modular Invariance, and Strings, Comm. Math. Phys. 106 (1986) 1.

[22] K.S.Narain, M.H.Sarmadi, E.Witten, A Note on Toroidal Compactification of Heterotic String Theory, Nucl. Phys. B279 (1987) 369.

[23] M.Rocek, E.Verlinde, Duality, Quotients, and Currents, Nucl. Phys. B373 (1992) 630, hep-th/9110053;

E.Bergshoeff, I.Entrop, R.Kallosh, Exact Duality in String Effective Action, Phys. Rev. D49 (1994) 6663, hep-th/9401025;

E.Alvarez, L.Alvarez-Gaume, I.Bakas, T-duality and Spacetime Supersymmetry, Nucl. Phys. B457 (1995) 3, hep-th/9507112. 
[24] O.Bergman, M.R.Gaberdiel, A Non-Supersymmetric Open String Theory and SDuality, Nucl. Phys. B499 (1997) 183, hep-th/9701137;

Y.Michishita, D0-branes in SO(32) $\times S O(32)$ Open Type 0 String Theory, Phys.Lett. B466 (1999) 161, hep-th/9907094.

[25] J.D.Blum, K.R.Dienes, Duality without Supersymmetry: The Case of the SO(16) $\times$ SO(16) String, Phys. Lett. B414 (1997) 260, hep-th/9707148; Strong/Weak Coupling Duality Relations for Non-Supersymmetric String Theories, Nucl. Phys. B516 (1998) 83, hep-th/9707160.

[26] J,Polchinski, E.Witten, Evidence for Heterotic - Type I String Duality, Nucl. Phys. B460 (1996) 525, hep-th/9510169.

[27] M.Bianchi, A.Sagnotti, On the Systematics of Open String Theories, Phys. Lett. B247 (1990) 517;

A.Sagnotti, Some Properties of Open-String Theories, hep-th/9509080.

[28] S.Chaudhuri, G.Hockney, J.D.Lykken, Maximally Supersymmetric String Theories in $D<10$, Phys. Rev. Lett. 75 (1995) 2264, hep-th/9505054;

S.Chaudhuri, J.Polchinski, Moduli Space of CHL Strings, Phys. Rev. D52 (1995) 7168, hep-th/9506048.

[29] H.M.S.Coxeter, Integral caylay numbers, in Twelve geometric essays, Southern Illinois University Press. 